\title{
Incompletness of Lukasiewicz and Gödel logics
}

\author{
M.A.Malkov \\ Russian research center for artificial intelligence
}

\begin{abstract}
Every $k$-valued logic is defined by a model such that every logical formula has a truth-table. We suppose that every truth-table of the model must have a logical formula, too. Then the logic is complete. This is a very strong condition since every model has $k^{k^{n}} n$-ary truth-tables. Nevertheless complete logics exist. But Lukasiewicz and Gödel logics are not complete.
\end{abstract}

\section{LOGIC, ALGEBRA AND COMPLETENESS}

Every logic is a theory and every theory has a model. The model of a logic is an algebra. We name objects of a logic and its model formulas and functions respectively.

A formula is a propositional variable or several propositional variables separated by connectivities $\vee, \wedge$ and $\neg$. The connective $\neg$ usually is replaced by a diacritic line. We will denote propositional variables by $p, p_{1}$, $p_{2}, \ldots$

Every function has a truth-table. We denote variables of functions by $x, x_{1}, x_{2}, \ldots$. The range of functions for $k$-valued logics is the set $\{0,1, \ldots, k-1\}$.

Every formula is interpreted by a function. It means that every formula has a truth-table. We call a logic complete, if every truth-table of the algebra has a formula of the logic.

Complete logics exist. For example, Post logic $P_{k}$ is complete [1]. Post proved this.

\section{LUKASIEWICZ LOGIC}

We shall prove that Lukasiewicz logic $L_{k}[2]$ is incomplete.

The logic has the next interpretations of its formulas.

Definition The formulas $p_{1} \vee p_{2}, p_{1} \wedge p_{2}$, and $\bar{p}$ are interpreted by the computable functions $\max \left(x_{1}, x_{2}\right), \min \left(x_{1}, x_{2}\right)$, and $k-1-x$.

By definition operations $\vee$ and $\wedge$ are idempotent, associative, commutative, absorbing and distributive. The operation $\neg$ has the next property: $\overline{\bar{p}}=p$

Theorem Lukasiewicz logic is incomplete.

Proof. Every algebra of a logic has $k^{k}$ one-ary functions. We shall prove that only 4 of them have formulas in the logic. These formulas are $p, \bar{p}, p \vee \bar{p}, p \wedge \bar{p}$. Indeed, using operations $\vee, \wedge$ and $\neg$ we have no new formula:

$$
p \vee(p \wedge \bar{p})=p, \bar{p} \vee(p \wedge \bar{p})=\bar{p}, p \wedge(p \vee \bar{p})=p, \bar{p} \wedge(p \vee \bar{p})=\bar{p}, \overline{p \vee \bar{p}}=p \wedge \bar{p}, \overline{p \wedge \bar{p}}=p \vee \bar{p}
$$

\section{GÖDEL LOGIC}

Gödel logic $\mathrm{G}_{k}$ [3] has the next interpretations of logical operations.

Definition The formulas $p_{1} \vee p_{2}, p_{1} \wedge p_{2}$, and $\bar{p}$ are interpreted by the computable functions $\max \left(x_{1}, x_{2}\right), \min \left(x_{1}, x_{2}\right)$, and $(k-1)(1-\operatorname{sg}(x))$, where $\operatorname{sg}(x)=0$ if $x=0$ and $\operatorname{sg}(x)=1$ if $x \neq 0$. 
By definition $\bar{x}=0$ if $x \neq 0$ and $\overline{0}=k-1$. Hear $\bar{x}=(k-1)(1-\mathrm{s} g(x))$. The other operations do not change.

Theorem Gödel logic is incomplete.

Proof. We shall prove that only 6 of one-ary functions have formulas in the logic. These formulas are $p$, $\bar{p}, \bar{p}, p \vee \bar{p}, p \wedge \bar{p}, \overline{p \wedge \bar{p}}$.

Indeed, using $\vee$ we have no new formula:

$$
\begin{aligned}
& p \vee \overline{\bar{p}}=\overline{\bar{p}}, \quad p \vee(p \vee \bar{p})=p \vee \bar{p}, \quad p \vee(p \wedge \bar{p})=p, \quad p \vee \overline{p \wedge \bar{p}}=\overline{p \wedge \bar{p}}, \\
& \bar{p} \vee \overline{\bar{p}}=\overline{p \wedge \bar{p}}, \quad \bar{p} \vee(p \vee \bar{p})=p \vee \bar{p}, \quad \bar{p} \vee(p \wedge \bar{p})=\bar{p}, \quad \bar{p} \vee \overline{p \wedge \bar{p}}=\overline{p \wedge \bar{p}} \\
& \overline{\bar{p}} \vee(p \vee \bar{p})=\overline{p \wedge \bar{p}}, \quad \overline{\bar{p}} \vee(p \wedge \bar{p})=\overline{\bar{p}}, \quad \overline{\bar{p}} \vee \overline{p \wedge \bar{p}}=\overline{p \wedge \bar{p}} \\
& (p \vee \bar{p}) \vee(p \wedge \bar{p})=p \vee \bar{p}, \quad(p \vee \bar{p}) \vee \overline{p \wedge \bar{p}}=\overline{p \wedge \bar{p}}, \quad(p \wedge \bar{p}) \vee \overline{p \wedge \bar{p}}=\overline{p \wedge \bar{p}}
\end{aligned}
$$

Using $\wedge$ we have no new formula, too:

$$
\begin{gathered}
p \wedge \overline{\bar{p}}=p, \quad p \wedge(p \vee \bar{p})=p, \quad p \wedge(p \wedge \bar{p})=(p \wedge \bar{p}), \quad p \wedge \overline{p \wedge \bar{p}}=p \\
\bar{p} \wedge \overline{\bar{p}}=p \wedge \bar{p}, \quad \bar{p} \wedge(p \vee \bar{p})=\bar{p}, \quad \bar{p} \wedge(p \wedge \bar{p})=p \wedge \bar{p}, \quad \bar{p} \wedge \overline{p \wedge \bar{p}}=\bar{p}, \\
\overline{\bar{p}} \wedge(p \vee \bar{p})=p, \quad \bar{p} \wedge(p \wedge \bar{p})=p \wedge \bar{p}, \quad \bar{p} \wedge \overline{p \wedge \bar{p}}=\overline{\bar{p}} \\
(p \vee \bar{p}) \wedge(p \wedge \bar{p})=p \wedge \bar{p}, \quad(p \vee \bar{p}) \wedge \overline{p \wedge \bar{p}}=p \vee \bar{p}, \quad(p \wedge \bar{p}) \wedge \overline{p \wedge \bar{p}}=p \wedge \bar{p}
\end{gathered}
$$

And using $\neg$ we again have no new formula:

$$
\overline{\bar{p}}=\bar{p}, \quad \overline{p \vee \bar{p}}=p \wedge \bar{p}, \quad \overline{p \wedge \bar{p}}=\overline{p \wedge \bar{p}}, \quad \overline{\overline{p \wedge \bar{p}}}=p \wedge \bar{p}
$$

\section{REFERENCES}

[1] Post E. L. Introduction to a general theory of elementary propositions. Amer. J. Math. (1921) 43.4, 163-185.

[2] Lukasiewicz, J. Selected Works. Amsterdam, North-Holland and Warsaw: PWN, 1970.

[3] Gödel K. Zum intuitionistischen Aussagenkalkül. Anz. Akad. Wiss. Wien (1932) 69, 65-66. 